\title{
Local concentrations
}

\author{
François Bavaud ${ }^{1}$ \\ ${ }^{1}$ University of Lausanne, Institute of Geography, Lausanne VD 1015, Switzerland \\ (e-mail: francois.bavaud@unil.ch)
}

Received: 13 September 2007 / Accepted: 13 May 2008

\begin{abstract}
Most global indices of concentrations are obtained as weighted averages of convex functions of distributions ratios, such as the per capita income. We seek to define local indices of concentrations, comparing the wealth of a region to its neighbours, where the spatial weights defining neighbourhood are formally equivalent to the components of a reversible Markov transition matrix. Second-order local concentrations are shown to generalize Moran or Geary autocorrelation indices, while first-order local concentrations can be constructed so as to not exceed their ordinary or global counterpart. Behaviour under aggregation and the Pigou-Dalton principle are further discussed within the proposed formalism, which is exemplified on wealth distribution among the Swiss cantons under the neighbourhood structure induced by interregional migrations.
\end{abstract}

\section{JEL classification: R12, C46, D31}

Key words: Concentration, spatial autocorrelation, local Gini index, Moran and Geary indices, neighbourhood

\section{Introduction}

Indices of concentrations measure the discrepancy between two distributions, typically between the regional income shares and the regional population shares. They constitute non-negative quantities attaining their minimum value zero when the two distributions coincide, decrease under aggregation, and obey the Pigou-Dalton transfer principle. Concentration indices, in particular spatial concentration of income and economic activites, have inspired an immense amount of empirical studies as well as applied mathematical literature; this scientific body is too huge to be faithfully reported, even shallowly (see however e.g., Atkinson 1970; Sen 1973; Cowell 1977; Yoshida 1977; Bourguignon 1979; Dagum 1980; Toyoda 1980; Shorrocks 1984; Baccini et al. 1986; Eichhorn 1988; Arbia 1989; Valeyre 1995).

The present paper initially describes a family of concentration indices covering most of the usual measures. As a matter of fact (Section 3), the usual concentration indices systematically turn out to express, as a weighted average of a convex function of the ratio of two distributions, where the average runs on regions (yielding first-order indices such as the Isard or the Theil 
concentrations) or on pairs of regions (yielding second-order indices such as the Gini or the quadratic concentrations). The study then focuses on the possible definitions and analytical properties of local concentrations indices, as measured from the neighbourhoods of the regions, in contrast to the usual global indices, where each region contributes in proportion of its relative population, irrespectively of its position.

Neighbourhood relations are quantified by the use of symmetric interaction of exchange matrices, or equivalently by row-normalized spatial weights defining the components of a reversible Markov transition matrix (see e.g., Bavaud 1998). This formalism encompasses most of the situations, such as those based upon a discrete adjacency matrix, a regular lattice or a continuous 'distance-deterrence function'. The same formalism also permits us to define in full generality the concept of spatial autocorrelation by comparing the values of the local to the global variances of a spatial variable.

In addition, this formalism naturally emphasizes an important property of ordinary, global concentrations, namely their decrease under local sharing, which is shown to entail both the decrease under aggregation and the Pigou-Dalton principles.

To a certain extent, the study of local concentrations generalizes and develops the study of local variances, central in the theory of spatial autocorrelation (see e.g., Moran 1950; Geary 1954; Lebart 1969; Haining 1990; Cressie 1993; Anselin 1995; Griffith 2000). Section 2 recalls the basic material on spatial autocorrelation, in the general context of reversible Markov chain or weighted networks, encompassing traditional approaches such as the use of adjacency matrices, of regular lattices or distance deterrence schemes (Bavaud 2007). Section 3 introduces the class of ordinary (global) first- and higher-order concentration indices under consideration. Section 4 studies the properties of various local concentration indices; in particular, the behavior of second-order local concentrations is analogous to spatial autocorrelation indices (Moran's $I$ and Geary's $c$ ), while first-order local concentrations, as defined here, are shown to be smaller than their global counterparts, and can be exactly decomposed under aggregation. Section 5 exemplifies the formalism with Swiss cantons' wealth data, with a neighbourhood structure induced by inter-regional migrations.

\section{The flow approach to spatial autocorrelation}

Consider $m$ regions exchanging units (people, goods, news) during some fixed time $T$, and define the normalized flow or exchange matrix $E$ with components $e_{i j}=$ 'proportion of units moving from region $i$ to region $j$ in time $T$ '. We assume here equilibrium (see Bavaud 2002, for a more general approach), that is the symmetry of $E$, as well as the strict positivity of the associated marginal or reference distribution $f$ :

$$
e_{i j} \geq 0 \quad e_{i j}=e_{j i} \quad f_{i}:=e_{i \bullet}=\sum_{j=1}^{m} e_{i j}>0 \quad e_{. .}=\sum_{i j} e_{i j}=\sum_{i} f_{i}=1
$$

$E$ defines a non-oriented weighted graph or network, possibly containing loops, together with a neighbourhood relation as ' $i \sim j$ ' iff $e_{i j}>0$. It also defines a reversible Markov chain with transition matrix $W=\left(w_{i j}\right)$ (with associated stationary distribution $f_{i}$ ) as

$$
w_{i j}:=\frac{e_{i j}}{f_{i}} \geq 0 \quad \sum_{j} w_{i j}=1 \quad \sum_{i} f_{i} w_{i j}=f_{j} \quad f_{i} w_{i j}=f_{j} w_{j i}=e_{i j} .
$$

In spatial econometrics, $W$ is the spatial weights matrix entering in spatially autoregressive models such as $y=\rho W y+X \beta+\varepsilon$ (see e.g. Anselin 1988). 

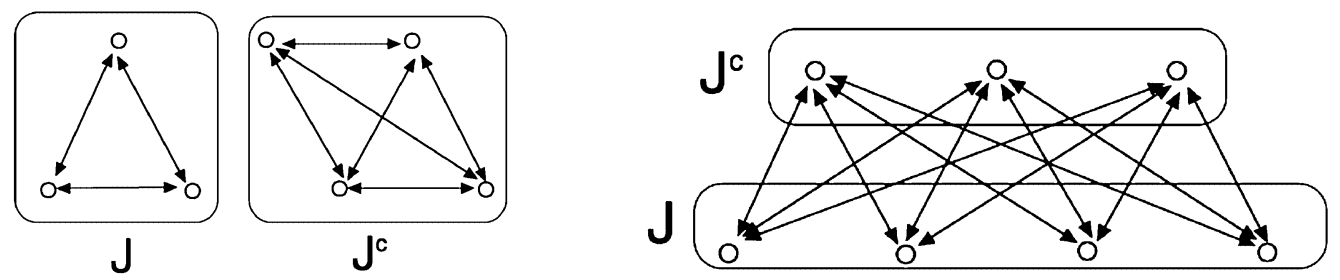

Fig. 1. Edges link pairs of neighbours in the above networks. Left: reducible network, with $\lambda_{2}=1$ and $e\left(J, J^{c}\right)=0$. Right: bipartite network, with $\lambda_{\mathrm{m}}=-1$ and $e\left(J, J^{c}\right)=1 / 2$

A regional variable $x$ is positively spatially autocorrelated if its local variance $\operatorname{var}_{\text {loc }}(x)$, measuring the average squared difference of values between pairs of neighbours, is smaller than the corresponding global or ordinary variance $\operatorname{var}(x)$, where pairs are chosen independently (see e.g. Lebart 1969; Anselin 1995; Bavaud 2007). Formally,

$$
\begin{aligned}
& \operatorname{var}_{\mathrm{loc}}(x):=\frac{1}{2} \sum_{i j} e_{i j}\left(x_{i}-x_{j}\right)^{2} \\
& \operatorname{var}(x):=\frac{1}{2} \sum_{i j} f_{i} f_{j}\left(x_{i}-x_{j}\right)^{2}=\sum_{i} f_{i}\left(x_{i}-\bar{x}\right)^{2}
\end{aligned}
$$

where $\bar{x}=\Sigma f_{i} x_{i}$. The ratio of the local variance (whose definition is reminiscent of the variogram construction) to the global variance is Geary's $c(x)$, obeying

$$
0 \leq 1-\lambda_{2} \leq c(x):=\frac{\operatorname{var}_{\mathrm{loc}}(x)}{\operatorname{var}(x)} \leq 1-\lambda_{m} \leq 2
$$

where $\lambda=1 \geq \lambda_{2} \geq \ldots \lambda_{m} \geq-1$ are the real eigenvalues of the reversible weight matrix $W$, where $\lambda_{1}=1$ is the trivial or Perron-Froebenius eigenvalue. The case $\lambda_{2}=1$ corresponds to a reducible network made of at least two non-interacting components, while $\lambda_{m}=1$ obtains for a bipartite network as illustrated in figure 1 (see e.g. Lawler and Sokal 1988; Chung 1997; Griffith 2000; Bavaud 2007).

Bipartite networks model pure commuting flows, where units are alternatively located in two complementary sets of regions, such as residences and workplaces.

Flows are said to be of a diffusive type if all the eigenvalues are non-negative. In this case, Geary's $c$ is bounded above by the unity, and Moran's index $I(x):=1-c(x)$ is non-negative. An extreme case of diffusive type is provided by frozen flows where $e_{i j}=f_{i} \delta_{i j}$ ( $\delta_{i j}$ being the components of the unit matrix), resulting in eigenvalues all equal to one as well as $c(x) \equiv 0$ and $I(x) \equiv 1$ for any field $x$. Frozen flows correspond to the absence of inter-regional exchange, that is complete autarchy.

To the opposite, perfect mobility case $e_{i j}=f_{i} f_{j}$ represents an a-spatial situation where each region sends the same proportion of units to the other regions, accordingly to the weight of the latter. The associated Markov chain is of order zero (memoryless transitions) and the eigenvalues, except $\lambda_{1}=1$, are zero. Under perfect mobility, local and global quantities coincide; in particular, $c(x) \equiv 1$ and $I(x) \equiv 0$.

\subsection{Low-diagonal transformation}

In the majority of spatial studies, the diagonal spatial weights are set to zero, which incidentally entails the existence of negative eigenvalues. However, zero diagonal values cannot in general be imposed to a given exchange matrix $E$ without modifying its eigenstructure. Even so, a 
low-diagonal exchange matrix $\tilde{E}=\left(\tilde{e}_{i j}\right)$ with the same marginals $\tilde{f}_{i}=f_{i}$ can be constructed as $\tilde{e}_{i j}:=e_{i j}+a\left(f_{i} f_{j}-f_{i} \delta_{i j}\right)$ where $a \geq 0$ must satisfy a $\leq a_{\max }:=\min _{i} e_{i i} /\left(f_{i}\left(1-f_{i}\right)\right)$ to insure the non-negativity of $\tilde{E}$ (see table 1$)$. The corresponding eigenvalues read $\tilde{\lambda}_{\alpha}=\lambda_{\alpha}+a\left(\delta_{\alpha 1}-1\right)$ : all the eigenvalues of $\tilde{W}$ with the exception of the trivial one are decreased by the value $a$. Also, one readily finds $\tilde{c}(x)=c(x)+a$ and $\tilde{I}(x)=I(x)-a$, which shows the local variance to increase under the low-diagonal transformation, due to the reduction of the self-interaction components $e_{i j}$ contributing to zero in Equation (1).

\section{Concentrations indices}

In the present, presumably original formalism (its global part included), concentration indices constitute asymmetric measures of discrepancy between any two distributions $f$ and $g$, the former being the reference distribution associated to an exchange matrix. For instance, the concentration between the reference distribution $f=$ 'population distribution' and $g=$ 'area distribution' is a measure of the variation of the population density across regions.

Wealth inequalities provide a well investigated, but far from being unique, illustration of the topics, where $f_{i}=$ 'share of the population of region $i$ ' is the reference distribution associated to inter-regional migrations in equilibrium, and $g_{i}=$ 'share of the income of region $i$ '. The ratio of the distributions is the income per capita, and constitutes a measure of regional wealth. The wealth concentration $Q$ is defined by

$$
Q:=\sum_{j} f_{j} c\left(q_{j}\right) \quad q_{j}:=\frac{g_{j}}{f_{j}}
$$

where $c(q)$, the concentration kernel, is convex with $c(l)=0$. Jensen's inequality (the average of a convex function is not less than the function of the average) together with $\bar{q}:=\Sigma f_{i} q_{i}=1$, demonstrates that $Q \geq 0$. In the equalitarian case $g \equiv f$, that is $q \equiv 1$, the concentration $Q$ is zero. Converserly, $Q=0$ implies the equalitarian wealth distribution when $c(q)$ is strictly convex.

Also, Equation (2) satisfies the Pigou-Dalton principle: if region $i$ transfers a part $\varepsilon>0$ of its income to region $j$, such that $g_{i} \rightarrow g_{i}-\varepsilon_{i}$ and $g_{i} \rightarrow g_{i}+\varepsilon_{i}$, the resulting concentration decreases by the quantity $\left(c^{\prime}\left(q_{i}\right)-\left(c^{\prime}\left(q_{j}\right)\right) \varepsilon+0\left(\varepsilon^{2}\right)\right.$, which is non-negative to the first order if $q_{i} \geq q_{j}$. Finally, the concentration decreases under aggregation: if regions are aggregated into superregions indexed by $J=1, \ldots, M<m$, then

$$
f_{J}:=\sum_{j \in J} f_{j} \quad g_{J}:=\sum_{j \in J} g_{j} \quad q_{J}:=\frac{g_{J}}{f_{J}}=\sum_{j \in J} \frac{f_{j}}{f_{J}} q_{j}
$$

and

$$
Q=\sum_{j} f_{j} c\left(q_{j}\right)=\sum_{J} f_{J} c\left(q_{J}\right)+\sum_{J} f_{J}\left(\sum_{j \in J} \frac{f_{j}}{f_{J}} c\left(q_{j}\right)-c\left(q_{J}\right)\right) \leq Q_{B}
$$

where the between concentration $Q_{B}:=\sum_{J} f_{J} c\left(q_{J}\right)$ and the within concentration $Q_{W}:=\sum_{J} f_{J}\left(\sum_{j \in J} \frac{f_{j}}{f_{J}} c\left(q_{j}\right)-c\left(q_{J}\right)\right)$ are both non-negative by Jensen's inequality. 


\begin{tabular}{|c|c|c|}
\hline & 2 & 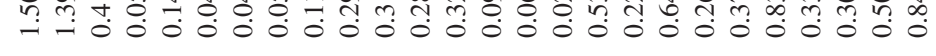 \\
\hline & 뜅 & 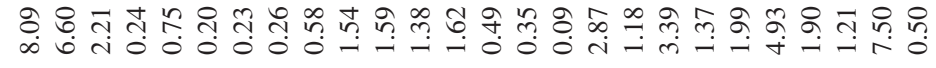 \\
\hline & 崩 & 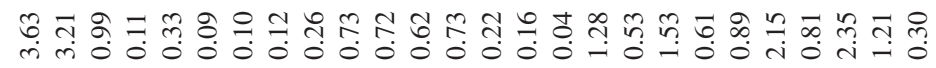 \\
\hline & $\stackrel{n}{>}$ & 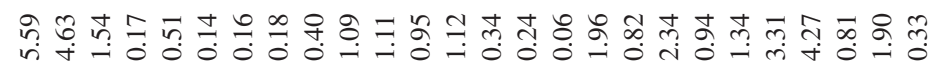 \\
\hline & 8 & 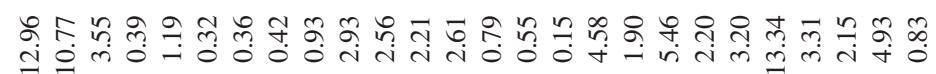 \\
\hline & $F$ & 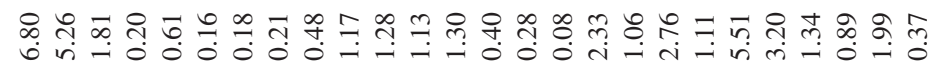 \\
\hline & 0 & 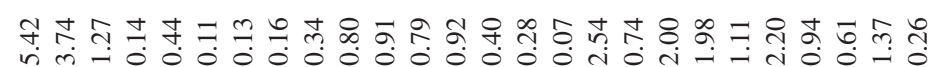 \\
\hline & U্ব & 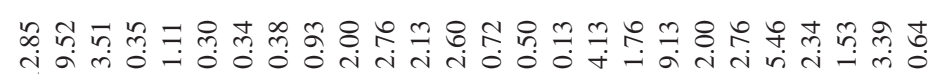 \\
\hline & 원 & 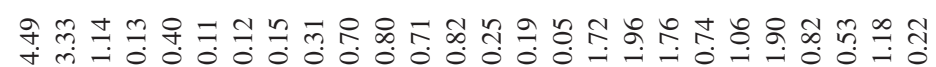 \\
\hline & U & 合令 \\
\hline & $\varangle$ & m \\
\hline 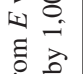 & 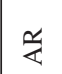 & 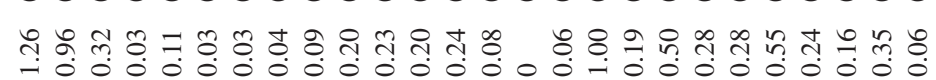 \\
\hline 吾 & $\overrightarrow{\mathbb{n}}$ & 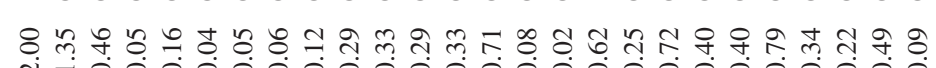 \\
\hline & & \\
\hline & $\vec{\otimes}$ & $a 7$ \\
\hline 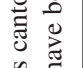 & $\tilde{\infty}$ & 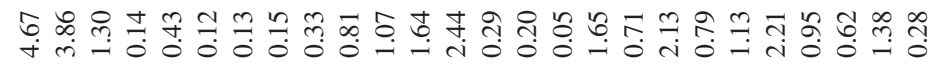 \\
\hline 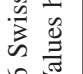 & O & 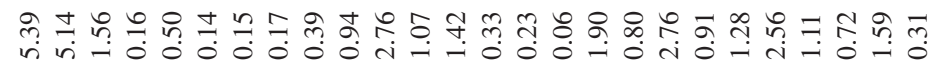 \\
\hline 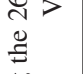 & $\frac{\alpha}{I}$ & 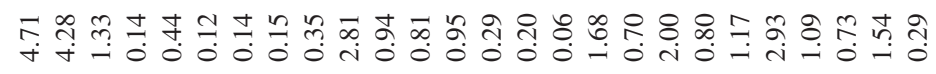 \\
\hline है & $\stackrel{\bigcup}{N}$ & 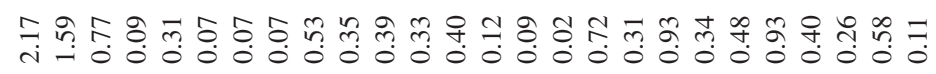 \\
\hline & ن & 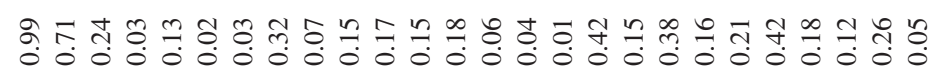 \\
\hline & 3 & 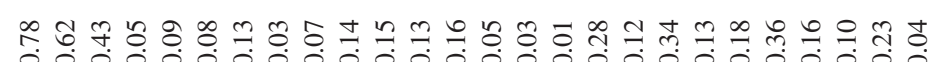 \\
\hline & & $a$ \\
\hline & o & क्. \\
\hline$\approx$ & N & 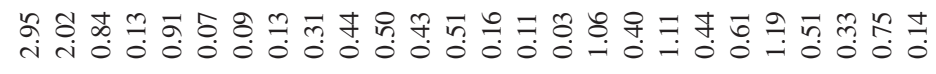 \\
\hline 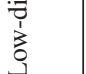 & 点 & 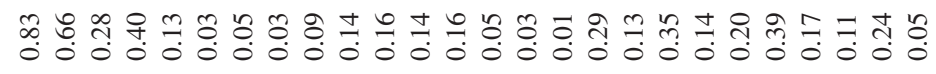 \\
\hline$\dot{-}$ & ב & 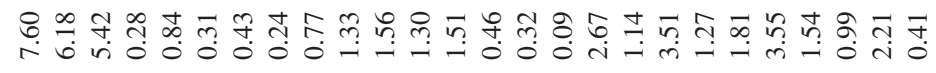 \\
\hline & 필 & 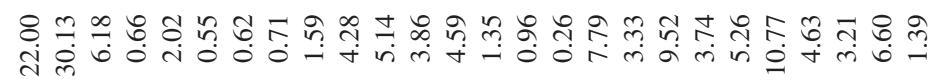 \\
\hline & $\stackrel{\mathbb{N}}{N}$ & 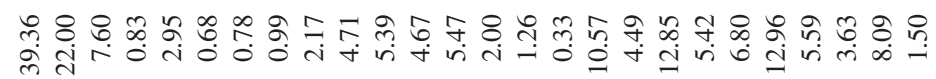 \\
\hline & & \\
\hline
\end{tabular}


Equation (2) encompasses many usual and possibly new concentration indices such as:

- the $L_{l}$ or Isard concentration with $c(q):=|q-1|$

- the quadratic or chi square concentration with $c(q)=(q-1)^{2}$

- the Theil or relative entropy concentration (Theil 1967; Gehrig 1988) with $c(q)=-\ln q$ and $Q=\Sigma f_{j} \ln \frac{f_{j}}{g_{j}}$

- the generalized entropy concentration kernel $c(q):=\frac{1}{\alpha(1-\alpha)}\left(q^{\alpha}-1\right)$ (see e.g. Cowell 1980), where

- $\alpha=2$ yields (half) the quadratic concentration

$\alpha \rightarrow 1$ yields the concentration $Q=\Sigma g_{j} \ln \frac{g_{j}}{f_{j}}$

$\alpha \rightarrow 0$ yields the Theil concentration

$\alpha=0.5$ yields (twice) the Hellinger concentration $Q=\Sigma\left(\sqrt{f_{j}}-\sqrt{g_{j}}\right)^{2}$

- the low threshold concentration kernel $c(q):=\theta(z-q)(z-q)$ where $z \leq 1$ defines a "poverty line' and $\theta(x)$ is the threshold function (taking on value 1 for $x \geq 0$ and value 0 for $x<0$ ). The resulting concentration is $Q=z F(z)-G(z) \geq 0$ where $F(z):=\Sigma f_{j} \theta\left(z-q_{j}\right)$ is the relative mass of poor regions and $G(z):=\Sigma g_{j} \theta\left(z-q_{j}\right)$ their corresponding income. Similar results obtain with the high threshold concentration kernel $c(q):=\theta(q-z)(q-z)$ where $z \geq 1$ represents an 'abundance threshold'.

\subsection{Decrease under local sharing}

The introduction of spatial weights permits to state an essential property shared by Equation (2), that is their decrease under local sharing: suppose each region donates its income to its neighbours, in proportion to the associated spatial weight, hence defining a new income $g^{[1]}$ and wealth $q^{[1]}$ :

$$
g_{j}^{[1]}:=\sum_{i} g_{i} w_{i j} \quad q_{j}^{[1]}:=\sum_{i} g_{i} \frac{w_{i j}}{f_{j}}=\sum_{i} g_{i} \frac{w_{j i}}{f_{i}}=\sum_{i} w_{j i} q_{i}
$$

Note that the shared wealth $q_{j}^{[1]}$, the average wealth of the neighbours of $j$, also satisfies $\Sigma f_{j} q_{j}^{[1]}=1$. As intuitively expected, the shared concentration $Q^{[1]}$ obeys $Q^{[1]} \leq Q$ :

$$
Q=\sum_{i} f_{i} c\left(q_{i}\right)=\sum_{j i} f_{j} w_{j i} c\left(q_{i}\right) \geq \sum_{j} f_{j} c\left(q_{j}^{[1]}\right)=: Q^{[1]}
$$

For a regular Markov chain, iterating the process further decreases the concentration with limit $Q^{[\infty]}=0$.

The concentration reduction under local sharing is a fairly general property, valid with any transition matrix $W$ such that $f$ is the associated stationary distribution. In particular, it implies the second law of thermodynamics in the Theil case, that there is a decrease of the relative entropy under neighbours averaging (see e.g., Cover and Thomas 1991). It also entails the decrease of concentration under aggregation, as well as the Pigou-Dalton principle themselves (see Appendix). 


\subsection{Concentrations of higher order}

The same properties hold for the concentration indices of order $r \geq 2$ defined: as averages over all $r$-uples of regions $\left(j_{1}, \ldots, j_{r}\right)$

$$
Q^{(r)}:=\frac{1}{r !} \sum_{j_{1}, \ldots, j_{r}} f_{j_{1}} \ldots f_{j r} \mathrm{c}\left(q_{j_{1}} \ldots, q_{j_{r}}\right)
$$

where the concentration kernel $c\left(q_{j_{1}}, \ldots, q_{j_{r}}\right)$ is a symmetric function, convex in each of its arguments, with $c(1, \ldots, 1)=0$. Equation (4), which does not seem to have been used in empirical studies, opens up the possibility of measuring concentration by comparing triples, quadruples, etc., of regions.

\section{Local concentrations}

\subsection{Local concentrations of order two}

In view of Equation (4), the global and local concentrations of order two are defined as:

$$
Q^{\mathrm{II}}:=\frac{1}{2} \sum_{i j} f_{i} f_{j} c\left(q_{i}, q_{j}\right) \quad Q_{\mathrm{loc}}^{\mathrm{II}}:=\frac{1}{2} \sum_{i j} e_{i j} c\left(q_{i}, q_{j}\right)
$$

where the kernel $c\left(q, q^{\prime}\right)$ is convex is both arguments and obeys $c(1,1)=0$. By construction, the global concentration decreases under local sharing:

$$
Q^{\mathrm{II}}=\frac{1}{2} \sum_{i j} f_{i} f_{j} c\left(q_{i}, q_{j}\right)=\frac{1}{2} \sum_{k l} \sum_{i j} f_{k} f_{l} w_{k i} w_{l j} c\left(q_{i}, q_{j}\right) \geq \frac{1}{2} \sum_{k l} f_{k} f_{l} c\left(q_{k}^{[1]}, q_{l}^{[1]}\right)=Q^{[1]} .
$$

It also gives $Q_{\mathrm{loc}}^{\mathrm{II}}=Q^{\mathrm{II}}$ under complete mobility. For frozen flows, $Q_{\mathrm{loc}}^{\mathrm{II}}=0$ under the additional condition $c(q, q)=0$.

The concentration of order two with quadratic kernel $c\left(q_{i}, q_{j}\right):=\left(q_{i}-q_{j}\right)^{2}$ is (as a result of the identity (1) for the global variance) identical with the corresponding concentration of order 1. The best known concentration index of order 2 is the Gini index with $c\left(q_{i}, q_{j}\right):=\left|q_{i}-q_{j}\right|$. Both are particular cases of the power concentration $c\left(q_{i}, q_{j}\right):=\left|q_{i}-q_{j}\right|^{p}$ with $p \geq 1$. Note that an admissible kernel such as $c\left(q_{i}, q_{j}\right)=\frac{q_{i}}{q_{j}} \ln q_{i}+\frac{q_{j}}{q_{i}} \ln q_{j}$ is not necessarily non-negative, nor satisfies $c(q, q)=0$.

However, definition (5) suffers from two drawbacks: to the best of our knowledge, a proof that $Q_{\mathrm{loc}}^{\mathrm{II}}$ decreases under aggregation and a proof that $Q_{\mathrm{loc}}^{\mathrm{II}} \leq Q^{\mathrm{II}}$ seem both lacking in general.

\subsubsection{Behaviour under aggregation}

Equation (5) can be written as:

$$
Q_{\mathrm{loc}}^{\mathrm{II}}=\sum_{i} f_{i} Q_{i}^{\mathrm{II}} \quad \text { where } \quad Q_{i}^{\mathrm{II}}:=\frac{1}{2} \sum_{j} w_{i j} c\left(q_{i}, q_{j}\right)
$$


The following decomposition holds under the aggregation of the $m$ regions into $M<m$ super-regions $\mathrm{J}=1, \ldots, M$ :

$$
Q_{i}^{I I}=\frac{1}{2} \sum_{J} w_{i J} c\left(q_{i}, q_{i}^{J}\right)+\frac{1}{2} \sum_{J} w_{j J} \sum_{j \in J} \frac{w_{i j}}{w_{j J}}\left[c\left(q_{i}, q_{j}\right)-c\left(q_{i}, q_{i}^{J}\right)\right]
$$

where $\quad w_{i J}:=\sum_{j \in J} w_{i j} \quad$ and $\quad q_{i}^{J}:=\sum \frac{w_{i j}}{w_{i J}} q_{j}$. By Jensen's inequality, $Q_{i}^{W}:=\frac{1}{2} \sum_{J} w_{i J} \sum_{j \in J} \frac{w_{i j}}{w_{i J}}$ $\left[c\left(q_{i}, q_{j}\right)-c\left(q_{i}, q_{i}^{J}\right)\right] \geq 0$ and $Q_{i}^{B} \geq \frac{1}{2} c\left(q_{i}, q_{i}^{[1]}\right)$, but a proof that $Q_{i}^{B} \geq 0$ is lacking for nonpositive kernels.

In the quadratic kernel case, the expression for $Q_{i}^{\mathrm{II}}$ (or rather its complementary, up to a normalization absorbing the denominator) turns out to coincide with the well-known local Moran statistic (Anselin 1995), used at detecting 'hot' and 'cold spots' in the configuration of a spatial field.

\subsubsection{Local versus global values}

$Q_{\mathrm{loc}}^{\mathrm{II}} \leq Q^{\mathrm{II}}$ does not hold in general. Indeed, in a world where rich regions are connected to poor regions only and vice-versa, the local concentration of order two can be as large as twice the global concentration: consider a bipartite graph with associated partition $\left(J, J^{c}\right)$ (see figure 1) such that $f_{J}=f_{J}^{c}=1 / 2$ and $e_{i j}=0$ if $(i, j) \in(J, J)$ or $(i, j) \in\left(J^{c}, J^{c}\right)$. Taking $q_{j}=1+\varepsilon$ for $j \in J$ and $q_{j}=1-\varepsilon$ for $\mathrm{j} \in J^{c}$ yields, for any kernel, $c\left(q, q^{\prime}\right)$,

$$
\frac{Q_{\mathrm{loc}}^{\mathrm{II}}}{Q^{\mathrm{II}}}=\frac{e\left(J, J^{c}\right)}{f_{J} f_{J}^{c}}=2 \text { where } e\left(J, J^{c}\right):=\sum_{i \in J, j \in J^{c}} e_{i j} .
$$

However, one can prove that $Q_{\mathrm{loc}}^{\mathrm{II}} \leq Q^{\mathrm{II}}$ for a diffusive network in the case of the power concentration for $p \leq 2$. The proof relies on the fact that the power $d_{i j}^{p / 2}$ of a Euclidean distance $d_{j i}$ is a Euclidean distance for $0 \leq p \leq 2$ (see e.g., Bretagnolle et al. 1966).

General graph bi-partitionning consists in determining a split into two regions $\left(J, J^{c}\right)$ with as little interacting as possible. The solution is also the minimum power concentration ratio for $p=1$ (see e.g., Deza and Laurent 1996), that is the minimum local Gini ratio:

$$
\frac{Q_{\mathrm{loc}}^{\mathrm{II}}}{Q^{\mathrm{II}}} \geq \min _{J, J^{C}} \frac{e\left(J, J^{c}\right)}{f_{J} f_{J^{c}}}=: \tilde{h}
$$

$\tilde{\mathrm{h}}$ is a so-called 'isoperimetric' constant (see e.g., Chung 1997), relating the 'surface' $e\left(J, J^{c}\right)$ to the 'volumes' $f_{J}$ and $f_{J^{c}}$. A variant is Cheeger's constant:

$$
h:=\min _{J, J^{c}} \frac{e\left(J, J^{c}\right)}{\min \left[f_{J}, f_{J^{c}}\right]}=\min _{q} \frac{\sum_{i j} e_{i j}\left|q_{i}-q_{j}\right|}{\min _{a} \sum_{i} f_{i}\left|q_{i}-a\right|}
$$

which trivially satisfies $\tilde{h} / 2 \leq h \leq \tilde{h}^{\prime}$ and obeys the celebrated Cheeger's inequalities $2 h \leq 1-$ $\lambda_{2} \leq 1-\sqrt{1-h^{2}}$ (see e.g., Chung 1997). 


\subsection{Local concentrations of order one}

Transforming the global first-order wealth concentration of order one Equation (2) into a local concentration is less immediate than in the second-order counterpart case of section 4.1.

\subsubsection{Version A}

A local individual wealth concentration $Q_{i}^{A}$ 'as observed from region $i$ ' can be defined by sampling the surroundings $j$ with weight $w_{i j}$, and rating their relative wealth as $q_{j} / q_{i}^{[1]}$, hence obtaining:

$$
Q_{i}^{A}:=\sum_{j} w_{i j} c\left(\frac{q_{j}}{q_{i}^{[1]}}\right) \quad Q_{\mathrm{loc}}^{A}:=\sum_{i} f_{i} Q_{i}^{A}=\sum_{i j} e_{i j} c\left(\frac{q_{j}}{q_{i}^{[1]}}\right)
$$

where $Q_{i}^{A} \geq 0$ by Jensen's inequality.

Under perfect mobility, $Q^{A}=Q$ by construction, and one would like $Q^{A} \leq Q$ to hold in the general case. However, a proof of the latter assertion seems lacking, with the notable exception of the Theil concentration $c(q)=-\ln q$, where one readily finds:

$$
Q_{\mathrm{loc}}^{A}=\sum_{j} f_{j} \ln \frac{g_{j}}{f_{j}}-\sum_{j} f_{j} \ln \frac{g_{j}^{[1]}}{f_{j}}=Q-Q^{[1]} \leq Q .
$$

\subsubsection{Version B}

Another attempt consists in defining a local wealth concentration as

$$
Q_{i}^{B}:=\sum_{j} w_{i j} c\left(q_{j}-q_{i}^{[1]}-1\right) \quad Q_{\mathrm{loc}}^{B}:=\sum_{i} f_{i} Q_{i}^{B}=\sum_{i j} e_{i j} c\left(q_{j}-q_{i}^{[1]}-1\right) .
$$

Again $Q_{i}^{B} \geq 0$, and $Q^{B}=Q$ under perfect mobility, but the statement $Q^{B} \leq Q$ remains unproved in general, with the exception of the quadratic concentration $c(q)=(q-1)^{2}$, where

$$
Q_{\mathrm{loc}}^{B}=\sum_{j} f_{j}\left(q_{i}-1\right)^{2}-\sum_{j} f_{j}\left(q_{i}^{[1]}-1\right)^{2}=Q-Q^{[1]} \leq Q
$$

\subsubsection{General attempt}

Equations (7) and (8) suggest a general definition for the individual local concentration $Q_{i}$ and the average local concentration of order one $Q_{\mathrm{loc}}$ :

$$
\begin{gathered}
Q_{i}:=\left[\sum_{j} w_{i j} c\left(q_{j}\right)\right]-c\left(q_{i}^{[1]}\right) \geq 0 \\
Q_{\mathrm{loc}}:=\sum_{i} f_{i} Q_{i}=\sum_{j} f_{j} c\left(q_{j}\right)-\sum_{j} f_{j} c\left(q_{j}^{[1]}\right)=Q-Q^{[1]} .
\end{gathered}
$$


The individual and average local concentrations enjoy a number of desirable properties:

- $0 \leq Q_{\mathrm{loc}} \leq Q$ (see section 3). In particular, $Q_{\mathrm{loc}}=0$ when $Q=0$ (equalitarian wealth).

- $Q_{\text {loc }}=0$ when the flows are frozen $\left(q^{[1]} \equiv q\right)$.

- $Q_{\text {loc }}=0$ when the flows are perfectly mobile: the distinction between local and global quantities vanishes in this case $\left(q^{[1]} \equiv 1\right)$.

- $Q_{i}$ and hence $Q_{\mathrm{loc}}$ decreases under aggregation of the $m$ regions into super-regions indiced by $J=1, \ldots, M<m$, in view of

$$
Q_{i}=\left[\sum_{J} w_{i J} c\left(q_{i}^{J}\right)\right]-c\left(q_{i}^{j}\right)+\sum_{J} w_{i J} \sum_{j \in J} \frac{w_{i j}}{w_{i J}}\left[c\left(q_{j}\right)-c\left(q_{i}^{J}\right)\right]
$$

where $\quad w_{i J}:=\sum w_{i j} \quad$ and $\quad q_{i}^{J}:=\sum_{j \in J} \frac{w_{i j}}{w_{i J}} q_{j}$. Again, $Q_{i}^{B}:=\left[\sum_{J} w_{i J} c\left(q_{i}^{J}\right)\right]-c\left(q_{i}^{j}\right) \geq 0 \quad$ and $Q_{i}^{W}:=\sum_{J} w_{i J} \sum_{j \in J} \frac{w_{i j}}{w_{i J}}\left[c\left(q_{j}\right)-c\left(q_{i}^{J}\right)\right] \geq 0$ by Jensen's inequality. Consequently

$$
Q_{\mathrm{loc}}=\sum_{i} f_{i} Q_{i}=\sum_{i} f_{i} Q_{i}^{B}+\sum_{i} f_{i} Q_{i}^{W}=: Q_{\mathrm{loc}}^{B}+Q_{\mathrm{loc}}^{W} \leq Q_{\mathrm{loc}}^{B}
$$

\section{Application: Inter-regional income disparities in Switzerland}

Consider the $m=26$ Swiss cantons, and the exchange matrix $E$ obtained from the interregional migration data 1995-2000 provided by the Swiss Federal Statistical Office (http:// www.bfs.admin.ch). The $(26 \times 26)$ origin-destination matrix $N=\left(n_{i j}\right)$ counts the number of individuals residing in canton $i$ in 1995 and in canton $j$ in 2000. $N$ is not symmetric, but is well approximated by a quasi-symmetric model (Bavaud 2002), and can be made symmetric by simply averaging with its transpose, or, as done here more sophisticated and explained in Bishop et al. (1975) and further developed by Bavaud (2002), by computing its maximum likelihood estimate under quasi-symmetry. After normalization, the resulting exchange matrix $E=\left(e_{i j}\right)$ induces a weighted neighbourhood structure, measuring the relative intensity of the migratory flow between each pair of cantons. The reference distribution $f_{i}=e_{i}$. yields the stationary population share of each canton, while the diagonal components $e_{i i}$ give the proportion of stayers, which strongly exceed the off-diagonal proportion of movers, most people staying in the same canton in the five years under consideration (Table 1).

The original non-trivial eigenvalues range from $\lambda_{2}=0.977$ to $\lambda_{26}=0.844>0$ : flows are of diffusive type, as expected from the time-continuous nature of the underlying migratory process. By contrast, low-diagonal eigenvalues with $a=a_{m}=0.863$ range from $\tilde{\lambda}_{2}=0.114$ to $\tilde{\lambda}_{26}=-0.018<0$.

Let $g_{i}=$ 'regional income share of the $i$-th canton in 2000' (http://www.bfs.admin.ch) constitute the second distribution. The resulting ratio $q_{i}=g_{i} / f_{i}$ is the per capita income in canton $i$ (see Figure 2 and Table 2).

Since the original and low-diagonal flows possess the same marginal $f$, the associated global concentration indices coincide. By contrast, the low-diagonal transformation reduces the regional self-interaction, especially strong in the original flows. Consequently, the individual low-diagonal local concentrations $\tilde{Q}_{j}$ are fairly larger than their original counterparts $Q_{\mathrm{i}}$, as shown by Table 2, irrespectively of the nature of the first- or second-order kernel under study. 


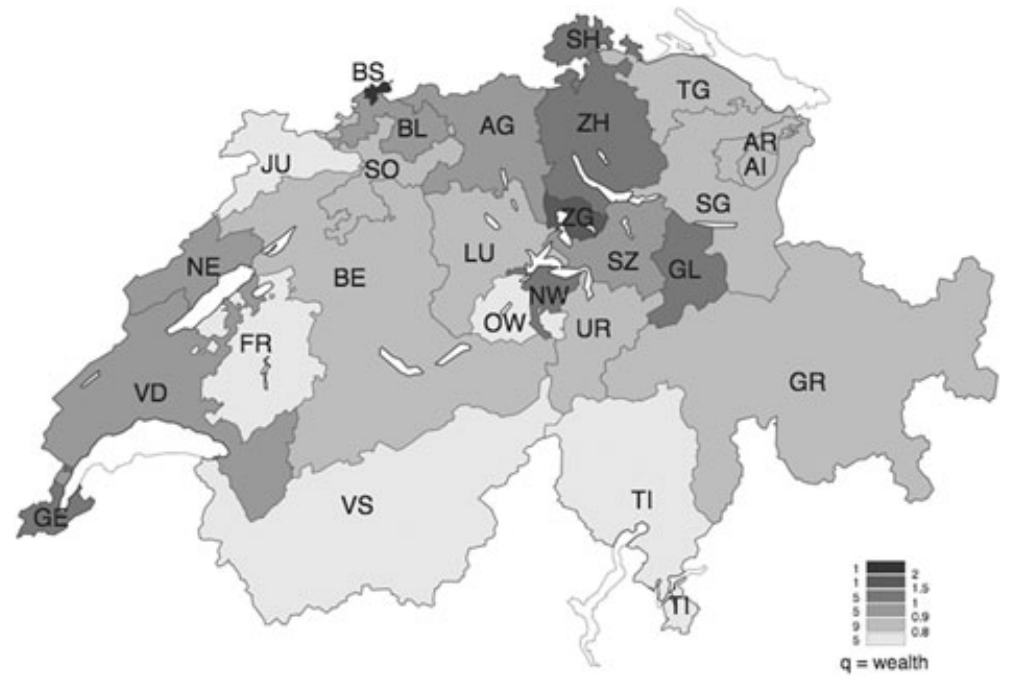

Fig. 2. Average wealth $q$ (per capita income) in the Swiss cantons, ranging from 0.704 (region VS) to 2.051 (region BS)

Note that $\left|q_{i}-1\right|>\left|\tilde{q}_{i}-1\right|$ (with the notable exception of $i=\mathrm{BL}$ ), which illustrates the regression of the shared wealth towards the mean. As a consequence, and as argued above, the local / global concentration ratios are less than unity. Indeed, Table 3 shows the ratios to be low, but this is mainly due to the strong auto-interaction, that is the high proportion of stayers in the data. Deleting a part of the stayers by the low-diagonal transformation increases the ratio near one. This follows from the weak auto-correlation of income distribution in Switzerland, which suggests that the diffusive, equalizing wealth effect, decreasing the local concentration, tends to be compensated by the preferential migrations effect from poorer to richer regions, increasing the local concentration.

\section{Conclusion}

In their ordinary, global version, first- and second-order concentration indices behave essentially in a similar way, and share the same desirable properties, such as the Pigou-Dalton principle and the exact decomposition under aggregation. This parallelism no longer holds in the local versions proposed in the present paper: on the one hand, local concentrations of order one, as defined in Equation (10), constitute presumably new objects possessing the sought-after properties of being smaller than their global counterparts, and exactly decomposable under aggregation. On the other hand, nothing comparable holds for second-order local concentrations which instead measure the local discontinuities of the wealth field, as does Geary's index, and allow the latter to be generalized to non-quadratic kernels. Although local concentrations of order two constitute extensions of classical Moran and Geary indices and permit fruitful connections with weighted graph theory (see e.g. Chung 1997), they essentially capture the same phenomenon, namely spatial autocorrelation.

The proximity between the numerical values of ratios of Table 3 comes as a bit of a surprise, and one could perhaps speculate over the possible robustness of the ratios, relatively insensitive to the choice of the concentration kernel. However, further analyses implying alternative data sets and spatial levels (such as networks of commuters, international migrations or international trade flow) are clearly requested to get a better and safer understanding of the possibilities and limitations offered by the present formalism. 


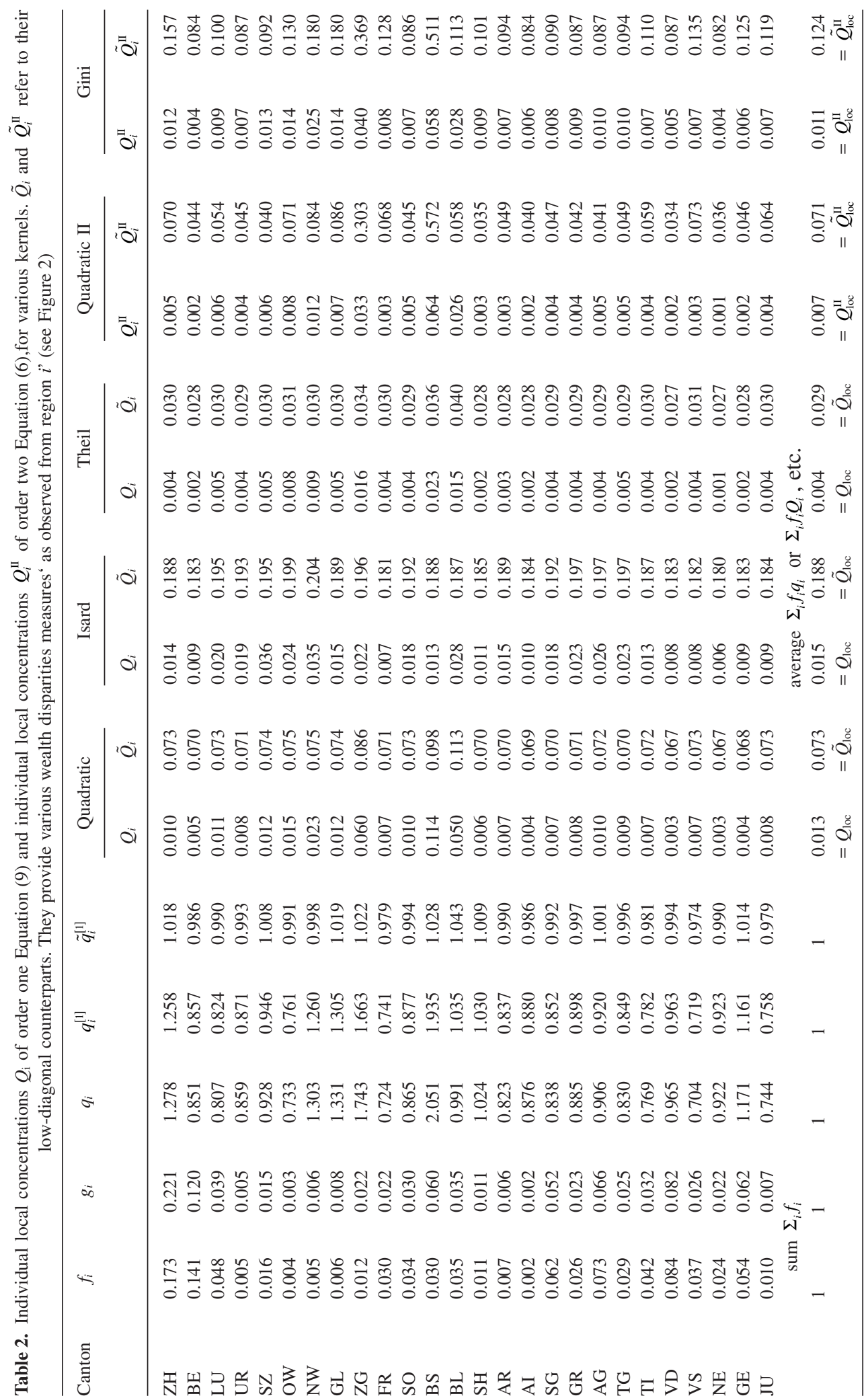


Table 3. Local versus global concentration ratios of order one and two, from Table 2

\begin{tabular}{|c|c|c|c|}
\hline Ratios of order one & Quadratic & Isard & Theil \\
\hline original flows $\frac{Q_{\mathrm{loc}}}{Q}$ & $\frac{0.013}{0.074}=0.174$ & $\frac{0.015}{0.202}=0.074$ & $\frac{0.004}{0.030}=0.151$ \\
\hline low-diagonal flows $\frac{\tilde{Q}_{\text {loc }}}{Q}$ & $\frac{0.073}{0.074}=0.996$ & $\frac{0.188}{0.202}=0.932$ & $\frac{0.029}{0.030}=0.996$ \\
\hline Ratios of order two & \multicolumn{2}{|c|}{ Quadratic II } & Gini \\
\hline original flows $\frac{Q_{\text {loc }}^{\mathrm{II}}}{Q^{\mathrm{II}}}$ & \multicolumn{2}{|c|}{$\frac{0.0068}{0.074}=0.092$} & $\frac{0.011}{0.131}=0.081$ \\
\hline low-diagonal flows $\frac{\tilde{Q}_{\mathrm{loc}}^{\mathrm{II}}}{Q^{\mathrm{II}}}$ & \multicolumn{2}{|c|}{$\frac{0.071}{0.074}=0.955$} & $\frac{0.124}{0.131}=0.944$ \\
\hline
\end{tabular}

\section{Appendix}

To show that Equation (3) entails the concentration decrease under aggregation, consider the transition matrix $w_{j k}:=I(J(j)=K(k)) f_{k} / f_{K(k)}$, where $J(j)$ denotes the super-region to which $j$ belongs, and $I(A)=1$ if $A$ is true, and $I(A)=0$ otherwise. $W$ is a Markov transition matrix with stationary distribution $f$ and $q_{j}^{[1]}=q_{J(j)}$, and hence $Q_{B}=Q^{[1]} \leq Q$.

Furthermore, let $q_{1}>q_{2}$ and $\varepsilon \in\left(0, \min \left[\left(q_{1}-q_{2}\right) f_{1},\left(q_{1}-q_{2}\right) f_{2}\right]\right)$. Define a Markov transition matrix $W$ as $w_{i j}=\delta_{i j}$ for $i, j \geq 3, w_{11}:=1-\lambda, w_{12}:=\lambda, w_{12}:=\mu$ and $w_{22}:=1-\mu$ (all other components being zero), where $\lambda:=\varepsilon /\left[\left(q_{1}-q_{2}\right) f_{1}\right]$ and $\mu:=\varepsilon /\left[\left(q_{1}-q_{2}\right) f_{2}\right]$. Then $W$ is a reversible transition matrix with stationary distribution $f$ and new income $g_{1}^{[1]}=g_{1}-\varepsilon, g_{1}^{(2)}=g_{2}+\varepsilon$, and $g_{i}^{[1]}=g_{i}$ for $i \geq 3$, hence showing the concentration to decrease under income transfer from a richer to a poorer region (Pigou-Dalton principle).

\section{References}

Anselin L (1988) Spatial econometrics. Kluwer, Dordrecht; Boston

Anselin L (1995) Local indicators of spatial association - LISA. Geographical analysis 27: 93-115

Arbia G (1989) Spatial data configuration in statistical analysis of regional economic and related problems. Kluwer, Dordrecht; Boston

Atkinson AB (1970) On the measurement of inequality. Journal of Economic Theory 2: 244-263

Baccini A, de Falguerolles A, Qannari EM (1986) Etude de quelques indices de concentration: Un essai de présentation unifiée. Revue de Statistique Appliquée 34: 31-44

Bavaud F (1998) Models for spatial weights: A systematic look. Geographical Analysis 30: 153-171

Bavaud F (2002) The quasi-symmetric side of gravity modelling. Environment and Planning A 34: 61-79

Bavaud F (2007) On spatial autocorrelation induced by exchanged flows. Submitted for publication

Bishop, YMM, Fienberg SE, Holland PW (1975) Discrete multivariate analysis. The MIT Press, Cambridge

Bourguignon F (1979) Decomposable income inequality measures. Econometrica 47: 901-920

Bretagnolle J, Dacunha Castelle D, Krivine J-L (1966) Lois stables et espaces L ${ }^{p}$. Annales de l'Institut Henri Poincaré 2: $231-259$

Chung FRK (1997) Spectral graph theory, CBMS Regional Conference Series in Mathematics, Vol. 92. American Mathematical Society, Providence, RI

Cover TM, Thomas JA (1991) Elements of information theory. Wiley, New York

Cowell FA (1977) Measuring inequality (1st edn.). Phillip Allan, Oxford

Cowell FA (1980) Generalized entropy and the measurement of distributional change. European Economic Review 13: $147-159$

Cressie NAC (1993) Statistics for spatial data. Wiley, New York

Dagum C (1980) Inequality measures between income distributions with applications. Econometrica 48: 1791-1803

Deza M, Laurent M (1996) Geometry of cuts and metrics. Springer-Verlag, Berlin

Eichhorn W (1988) On a class of inequality measures Social Choice and Welfare Vol.5, pp. 171-177 
Geary RC (1954) The contiguity ratio and statistical mapping. Incorporated Statistician 5: 115-145

Gehrig W (1988) On the Shannon-Theil concentration measure and its characterizations. In: Eichhorn W (ed) Measurement in Economics. Physica Verlag, Heidelberg

Griffith DA (2000) Eigenfunction properties and approximations of selected incidence matrices employed in spatial analyses. Linear Algebra and its Applications 321: 95-112

Haining RP (1990) Spatial data analysis in the social and environmental sciences. Cambridge University Press, Cambridge, MA

Lawler GF, Sokal AD (1988) Bounds on the $L^{2}$ spectrum for Markov chains and Markov processes: A generalization of Cheeger's inequality. Transactions of the American Mathematical Society 309: 557-580

Lebart L (1969) Analyse statistique de la contiguïté. Publication de l'Institut de Statistiques de l'Université de Paris 28 : $81-112$

Moran PAP (1950) Notes on continuous stochastic phenomena. Biometrika 37: 17-23

Sen AK (1973) On economic inequality. Clarendon Press, Oxford

Shorrocks AF (1984), Inequality decomposition by population subgroups. Econometrica 52: 1369-1385

Theil H (1967) Economics and information theory. North Holland, Amsterdam

Toyoda T (1980) Decomposability of inequality measures. Economic Studies Quarterly 31: 207-246

Valeyre A (1995) Formes et propriétés des indices d'inégalité entre proportions. Mathématiques et Sciences Humaines 132: 13-37

Yoshida T (1977) The necessary and sufficient condition for additive separability of income inequality measures. Economic Studies Quarterly 28: 160-163 


\title{
Concentraciones locales
}

\author{
François Bavaud
}

Resumen. La mayoría de índices globales de concentraciones se obtienen como promedios ponderados de funciones convexas de índices de distribución, como la renta per capita. Buscamos definir índices locales de concentraciones, comparando la riqueza de una región con sus vecinas, donde las ponderaciones espaciales que definen la vecindad son formalmente equivalentes a los componentes de una matriz de transición Markov reversible. Se muestran concentraciones locales de segundo orden para generalizar los índices de autocorrelación de Moran o Geary, mientras que las concentraciones locales de primer orden se elaboran de manera que no excedan su contraparte ordinaria o global. Se discuten el comportamiento bajo agregación y el Principio de Pigou-Dalton dentro del formalismo propuesto, el cual se ejemplifica mediante la distribución de riqueza entre cantones suizos bajo la estructura de vecindad inducida por migraciones regionales.

\section{JEL classification: R12, C46, D31}

Palabras clave: Concentración, autocorrelación espacial, índice de Gini local, índices de Moran y Geary, vecindad

要旨：１人当たり所得のような世界的な集中度指標の大部分は、分布指数の加重平均凸関数として求め られる。我々は、地域の富裕度を近隣地域と比較して、地域的な集中度指標の定義を求める。そこでは、 近隣を定義する空間的重みは、可逆的マルコフ遷移マトリクスの要素と形式的に等しい。Moran または Geary の自己相関指数を一般化するために二次地方集中が示され、その一方で、通常のあるいは世界にお ける比較対象を超えないよう、一次地方集中を構築することが可能である。集団及び Pigou-Dalton の原 則下での行動はさらに提示形式内で検証され、それは相互地域移動によって生じた近隣構造下でのスイ スの州間における富の配分で例示される。 